\title{
Selective Capture of Magnetic Wires to Particles in High Gradient Magnetic Separation
}

\author{
Jianwu Zeng, Xiong Tong *, Fan Yi and Luzheng Chen *(1) \\ Faculty of Land Resource Engineering, Kunming University of Science and Technology, Kunming 650093, China \\ * Correspondence: xiongtong2000@yahoo.com (X.T.); chluzheng@kmust.edu.cn (L.C.); \\ Tel.: +86-871-6518-0062 (L.C.)
}

Received: 30 June 2019; Accepted: 21 August 2019; Published: 23 August 2019

\begin{abstract}
High gradient magnetic separation (HGMS) achieves effective separation to fine weakly magnetic minerals using numerous small magnetic wires in matrix, and its separation performance is inherently dependent on the capture characteristics of the wires. In this work, the selective capture of magnetic wire to particles in high gradient magnetic field was theoretically described and simulated using COMSOL Multiphysics. It was found that the capture trajectories of a small amount of particles under the ideal condition was significantly different from those of a large amount of particles under the actual condition, and non-magnetic particles would be much more easily entrained into magnetic deposits captured onto the wire surface under the actual condition than those under the ideal condition. These theoretical and simulated results were basically validated with the experimental magnetic capture to an ilmenite ore, and the wires in slow feed mode have achieved much higher capture selectivity than those in the fast feed mode. For instance, at the magnetic induction of $0.8 \mathrm{~T}$, the $\mathrm{TiO}_{2}$ grade of magnetic deposits captured by $3 \mathrm{~mm}$ diameter wire in the slow feed model reached $36.78 \%$, which is higher than $28.32 \%$ in the fast feed model. The selective capture difference between the fast and slow feed models increased with increase in the magnetic induction and with decrease in the pulsating frequency. This investigation contributes to improve HGMS performance in concentrating fine weakly magnetic ores.
\end{abstract}

Keywords: high gradient magnetic separation; capture selectivity; magnetic wire; matrix

\section{Introduction}

High gradient magnetic separation (HGMS) is an effective method for the concentration or removal of paramagnetic particles from various suspensions [1], and it has been widely applied in the field of mineral processing, for recovery of fine weakly magnetic minerals and for removal of such minerals from non-metallic ores [2,3]. This method is achieved through the use of numerous small magnetic wires in matrix, to generate a high magnetic field gradient at the vicinity of the wires in background magnetic field, and consequently produce a sufficiently powerful magnetic capture force to magnetic particles [4]. Therefore, the HGMS performance is inherently dependent on the capture characteristics of the wires [5]. But, as we know, in the most cases HGMS separators were basically limited in the roughing or scavenging process of weakly magnetic ores such as hematite and ilmenite $[6,7]$, as they produced relatively low-grade concentrates. Obviously, the low-grade concentrates were resulted from the relatively low capture selectivity of magnetic wires to magnetic particles in the HGMS separators.

Theoretically, magnetic particles would be captured onto the wire due to the action of magnetic capture force, while non-magnetic particles would flow through the wire. However, in practice, some non-magnetic particles will be inevitably entrained into magnetic deposits in HGMS process, reducing the capture selectivity of the wire. The significant deviation of practical wire capture from the theoretical one has attracted much attention over the years, and many people have reported the 
capture selectivity of magnetic wire to particles in HGMS process. For instance, it has been early reported that the low separation selectivity of HGMS is mainly resulted from the non-looseness of slurry flowing around the wire, and thus pulsating HGMS technology was invented; in this new technology, the pulsating slurry improves the looseness of slurry, and significantly enhances the HGMS selectivity [8]. Ren et al. has comparatively investigated the capture selectivity of single wire and multi-wires, and found that the single wire achieved obviously superior selectivity to that of the multi-wires [9]. Moreover, some other researchers reported the dependence of capture selectivity on the shape of magnetic wire [10,11]. The build-up of magnetic particles onto magnetic wire, the size matching of magnetic wire to independent particles and the capture dynamics of magnetic wire in fluid were also reported, in order to understand the basic principles of wire magnetic capture in HGMS process [12-14].

These investigations are encouraging, as they contributed to improve HGMS performance. However, the inherent and detailed entraining mechanism of non-magnetic particles into magnetic deposits on a magnetic wire surface is insufficient, which has restricted the development of HGMS technologies. In this work, the selective capture of magnetic wire to particles in high gradient magnetic field was theoretically described and simulated using COMSOL Multiphysics. These theoretical and simulated results were then basically validated with the experimental magnetic capture to ilmenite ore, using an experimental magnetic capture method.

\section{Theoretical}

\subsection{Magnetic Capture of Wire to Particles}

As shown in Figure 1, suppose a cylindrical magnetic wire of diameter $a$ is placed in the separating zone of a HGMS process and a uniform magnetic field $B_{0}$ is applied in the zone. A paramagnetic magnetic particle of volume $V$ and magnetic susceptibility $K$ is carried through the wire by slurry flowing at a velocity of $v_{0}$. It is noted that the slurry is parallel to the applied filed and they are perpendicular to the axis of wire.
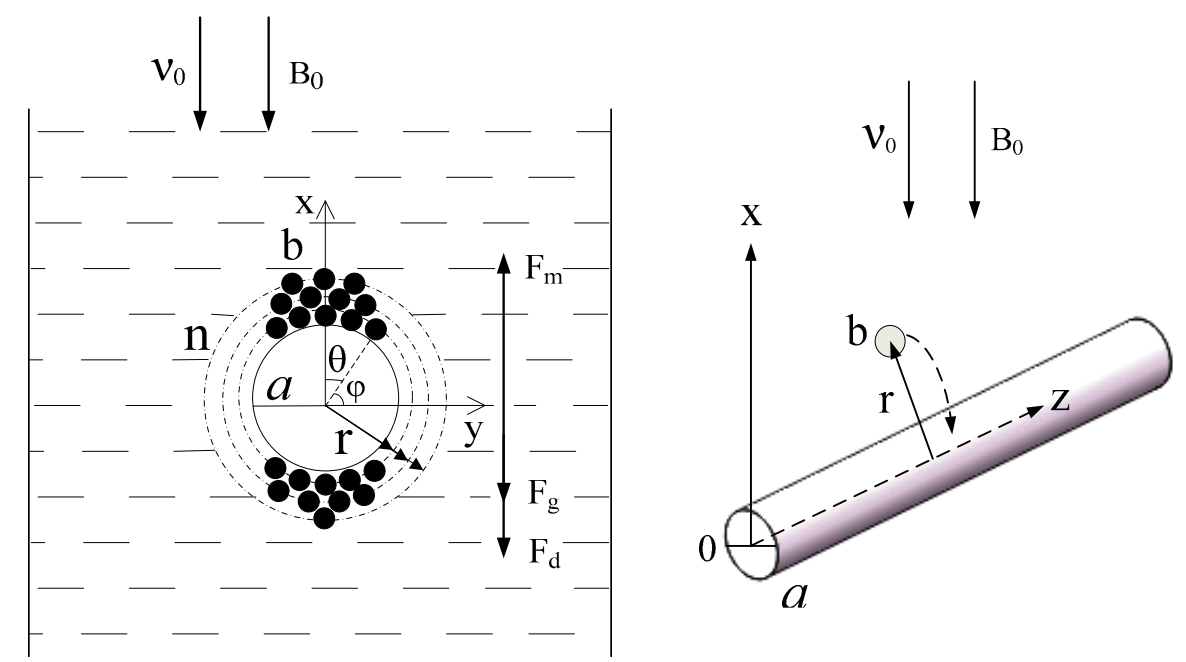

Figure 1. High gradient magnetic separation (HGMS) process (left) and magnetic capture of cylindrical wire to magnetic particle (right).

In the above process, the dominating forces that should be considered are magnetic force $F_{m}$, hydrodynamic drag $F_{d}$ and gravity force $F_{g}$. 


\subsubsection{Magnetic Force}

The magnetic force acting onto the particle is proportional to the magnetic induction and gradient, as shown in Equation (1).

$$
F_{m}=\frac{1}{6 \mu_{0}} \pi K b^{3} \operatorname{BgradB},
$$

where, $\mu_{0}$ is the permeability of free space, $b$ is the diameter of particle.

In the polar coordinate $\mathrm{P}(\mathrm{r}, \theta)$, Equation (1) is further written as:

$$
\left\{\begin{array}{l}
F_{m r}=\frac{\pi}{12 \mu_{0}} K b^{3} B_{0}{ }^{2} \frac{a^{2}}{r^{3}}\left(\frac{a^{2}}{4 r^{2}}+\cos 2 \theta\right) \\
F_{m \theta}=\frac{\pi}{12 \mu_{0}} K b^{3} B_{0} \frac{a^{2}}{r^{3}} \sin 2 \theta
\end{array},\right.
$$

where, $r$ is the capture radius of magnetic force from the center of wire to that of particle.

\subsubsection{Hydrodynamic Drag}

The hydrodynamic drag is the main force against the capture of magnetic force, and it could be calculated using Stokes formula:

$$
F_{\mathrm{d}}=3 \pi \eta b\left(v_{l}-v_{p}\right),
$$

where, $v_{l}$ and $v_{p}$ are the velocity of slurry and particles, respectively.

Suppose the slurry is inviscid and incompressible, and then the velocity of slurry flowing through the wire could be calculated using Equation (4):

$$
\left\{\begin{array}{l}
v_{r}=v_{0} \cdot\left(1-\frac{a^{2}}{4 r^{2}}\right) \cos \theta \\
v_{\theta}=-v_{0} \cdot\left(1+\frac{a^{2}}{4 r^{2}}\right) \sin \theta
\end{array}\right.
$$

Combing Equations (3) and (4), the hydrodynamic drag could be further written as:

$$
\left\{\begin{array}{l}
F_{d r}=3 \pi \eta b\left[v_{0} \cdot\left(1-\frac{a^{2}}{4 r^{2}}\right) \cos \theta-\frac{d_{r}}{d_{t}}\right] \\
F_{d \theta}=3 \pi \eta b\left[-v_{0} \cdot\left(1+\frac{a^{2}}{4 r^{2}}\right) \sin \theta-r \frac{d_{r}}{d_{0}}\right]
\end{array},\right.
$$

where, $\frac{d_{r}}{d_{t}}$ and $r \frac{d_{\theta}}{d_{t}}$ are the radial component and tangential components of particle velocity.

\subsubsection{Gravitational Force}

The effective gravitational force acting onto the particle is:

$$
\left\{\begin{array}{l}
F_{\mathrm{g} r}=\left(\rho_{p}-\rho_{f}\right) g V \cos \theta \\
F_{\mathrm{g} \theta}=\left(\rho_{p}-\rho_{f}\right) g V \sin \theta
\end{array}\right.
$$

where, $\rho_{p}$ and $\rho_{f}$ are the density of particle and fluid, respectively; $g$ is the gravitational acceleration.

\subsubsection{Required Magnetic Force for Effective Capture}

For a particle to be effectively captured onto the wire, the interactive force between magnetic wire and particle should be attractive, i.e., magnetic force counteracts competing force:

$$
\left\{\begin{array}{l}
F_{m r} \geq F_{d \theta}+F_{g r} \\
F_{m \theta} \geq F_{d \theta}+F_{g \theta}
\end{array},\right.
$$




\subsection{Motion Trajectory of Particle}

\subsubsection{Trajectory of Independent Particles}

The motion of particles is determinable by Newton's second law:

$$
\vec{F}=m \vec{a},
$$

where, $\vec{F}$ is the resultant force acting onto the particle, $m$ and $\vec{a}$ are the mass and accelerated velocity of particle, respectively.

For an independent particle, the interactions between the particle and slurry, the van der Waals force and the interactions between particles is negligible, so the trajectory of particle is mainly determined by the magnetic force, hydrodynamic drag and gravitational force:

$$
\frac{d\left(m_{p} v\right)}{d t}=\vec{F}_{\mathrm{d}}+\vec{F}_{\mathrm{m}}+\vec{F}_{\mathrm{g}}
$$

Combing Equations (3), (5), (6), and (9), the motion equation of the independent particle was obtained:

$$
\left\{\begin{array}{l}
\frac{d_{r}}{d_{t}}=\frac{2 v_{0}}{a}\left(1-\frac{a^{2}}{4 r^{2}}\right) \cos \theta-\frac{8 K B_{0}^{2} b^{2}}{9 \eta \mu_{0} a^{2}}\left(\frac{1}{r^{5}}+\frac{\cos 2 \theta}{r^{3}}\right) \\
r \frac{d_{\theta}}{d_{t}}=-\frac{2 v_{0}}{a}\left(1+\frac{a^{2}}{4 r^{2}}\right) \sin \theta-\frac{8 K B_{0}^{2} b^{2}}{9 \eta \mu_{0} a^{2}} \cdot \frac{\sin 2 \theta}{r^{3}}
\end{array},\right.
$$

Based on Equation (10) and the COMSOL Multiphysics simulation, the trajectories of independent particles are shown in Figure 2, and the related boundary conditions for the simulation are listed in Table 1. It is noted that in the simulation, only six magnetic particles and six non-magnetic particles were fed into the separating zone from the inlet at the initial time, so that the interactions between the particles could be neglected in the simulation; in addition, the interactions between the particles and slurry are not considered in the simulation.

Table 1. Boundary conditions for COMSOL Multiphysics simulation.

\begin{tabular}{cc}
\hline Boundary Conditions & Set Value \\
\hline Relative magnetic permeability of slurry & 1 \\
Relative magnetic permeability of wire & 7000 \\
Background magnetic induction & 0.6 and $1.0 \mathrm{~T}$ \\
Viscosity of slurry & $1.2 \times 10^{-3} \mathrm{~Pa} \cdot \mathrm{s}$ \\
Density of slurry & $1.3 \times 10^{3} \mathrm{~kg} / \mathrm{m}^{3}$ \\
Volume magnetic susceptibility of particle & $2.57 \times 10^{-3}$ \\
Density of particle & $4.5 \times 10^{3} \mathrm{~kg} / \mathrm{m}^{3}$ \\
Diameter of particle & $0.10 \mathrm{~mm}$ \\
Feed velocity & $0.04,0.06$ and $0.10 \mathrm{~m} / \mathrm{s}$ \\
\hline
\end{tabular}

From Figure 2, the trajectories of magnetic particles at the initial time are similar to those of non-magnetic particles, as they are mainly dragged by the hydrodynamic drag during this period. However, when the particles move around the wire, the trajectories of magnetic particles are significantly different from those of non-magnetic particles. The magnetic particles would be attracted by the magnetic wire due to the action of magnetic force, resulting in their variation in their trajectories. But, when the non-magnetic particles move around the wire, they flow through the wire under the action of hydrodynamic drag, with two non-magnetic particles colliding with the wire due to their inertia effect under the particular condition of $1.2 \mathrm{~T}$ magnetic induction and $3.0 \mathrm{~mm} / \mathrm{s}$ flow velocity; but, they were instantaneously bounced back by the magnetic wire. 
It can be seen from the above discussion that under the ideal condition, the magnetic wire in the magnetic field only captures magnetic particles, and the non-magnetic particles would not be captured onto the magnetic wire as a result of the hydrodynamic drag.
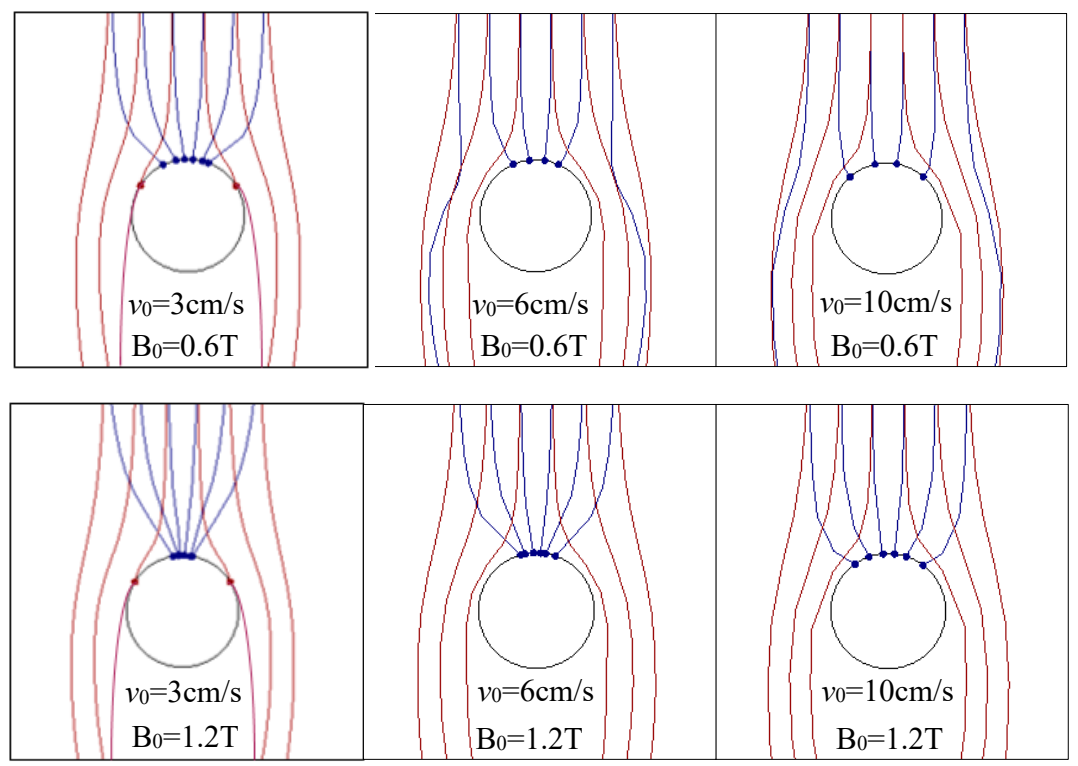

$\rightarrow$ Magnetic particle $\quad \rightarrow$ Non-magnetic particle

Figure 2. Simulated trajectories of independent particles in capture of single-wire.

\subsubsection{Trajectory of Interactive Particles}

In the actual HGMS process, the interactions between particles, and those between particles and slurry, have definitely their effects on the separation performance, due to the fact that the particles are fast and massively fed into the separating zone of HGMS separator. These interactions should be considered in the calculation and simulation for the motion trajectories of particles:

$$
\frac{d\left(m_{p} v\right)}{d t}=\vec{F}_{\mathrm{d}}+\vec{F}_{\mathrm{m}}+\vec{F}_{\mathrm{g}}+\vec{F}_{\mathrm{p}}+\vec{F}_{\mathrm{s}}
$$

where, $\vec{F}_{\mathrm{p}}$ and $\vec{F}_{\mathrm{s}}$ are the interactions between particles, and those between particles and slurry, respectively.

The interactions between the particles in Equation (11) could be written as:

$$
\vec{F}_{\mathrm{p}}=\frac{A}{3}\left[\frac{2 b^{2}(x+2 b)}{\left(x^{2}+4 b x\right)^{2}}+\frac{2 b^{2}}{(x+2 b)^{2}}-\frac{x+2 b x}{x+4 b}+\frac{1}{x+2 b^{\prime}}\right.
$$

where, $A$ is the constant of Hamaker, and $x$ is the distance between adjacent particles.

Also, the interactions between particles and slurry could be directly calculated using the COMSOL Multiphysics 5.4 .

Using the same boundary conditions (Table 1) as those for the simulation independent particles, the simulated trajectories of interactive particles are shown in Figure 3. It is noted that in the simulation the particles are continuously and massively fed into the separating zone.

Combing Figures 2 and 3, it is clear that the trajectories of interactive particles are significantly different from those of independent particles. As can be seen in Figure 3, most of non-magnetic particles directly flow through the wire, but a small part of the particles would be entrained into the magnetic deposit captured on the wire surface. These particles could not be released from the deposits under the action of interactions between adjacent particles, which results in the reduced capture selectivity 
of the wire. It was found from Figure 3 that under the higher magnetic induction and lower slurry velocity, the non-magnetic particles would be more easily entrained into the magnetic deposits on the wire surface.

In addition, the interactive particles were captured onto the wire in multi-layers, due to the fact that these particles were continuously and massively fed into the zone. In this multi-layer capture, the particles entrained into the inner layers would be wrapped by outer particles, leading to the difficulty for releasing the entrained particles. It is noted that the particles in multi-layers form a dense deposit on the wire surface under the action of strong magnetic force and the interactions between particles, preventing the entrained particles to be released from the deposits.
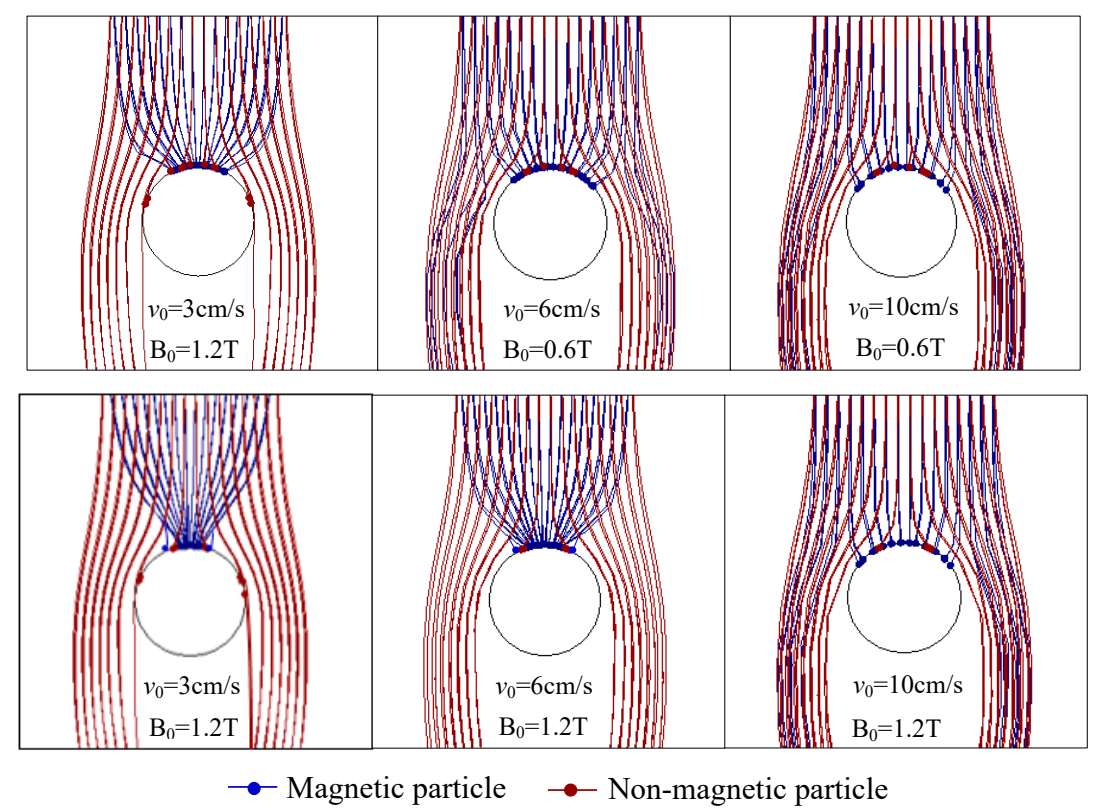

Figure 3. Simulated trajectories of interactive particles in capture of single-wire.

\subsection{Fluid Characteristics around Single-Wire and Multi-Wires}

In practice, matrix is made of numerous magnetic wires, so that a comparative investigation on the magnetic capture of single-wire and multi-wires would provide a crucial foundation for improving HGMS performance. It is noted that the difference in the capture characteristics of single-wire and multi-wires in the HGMS process is mainly resulted from their different fluid characteristics around the wires. Therefore, in this section, the fluid characteristics around the single-wire and multi-wires were respectively simulated using the COMSOL Multiphysics, as shown in Figure 4.

From Figure 4, for the single wire, there is a roundabout flow around the wire, and the slurry flows out of the wires, contributing to release the non-magnetic particles. However, after the particles are entrained in the upstream and downstream of the wire surface, the flow velocity of slurry on these two areas is nearly zero, increasing the difficulty for releasing the non-magnetic particles. For the multi-wires, the slurry flowing around the wire would be affected by adjacent wires, and it cannot flow through the wires freely. Comparing the fluid characteristics around the single-wire and multi-wires, the flowability of slurry in the multi-wires is much weaker than that through the single-wire, so that the capture selectivity of multi-wires gets lower than that of the single-wire. 

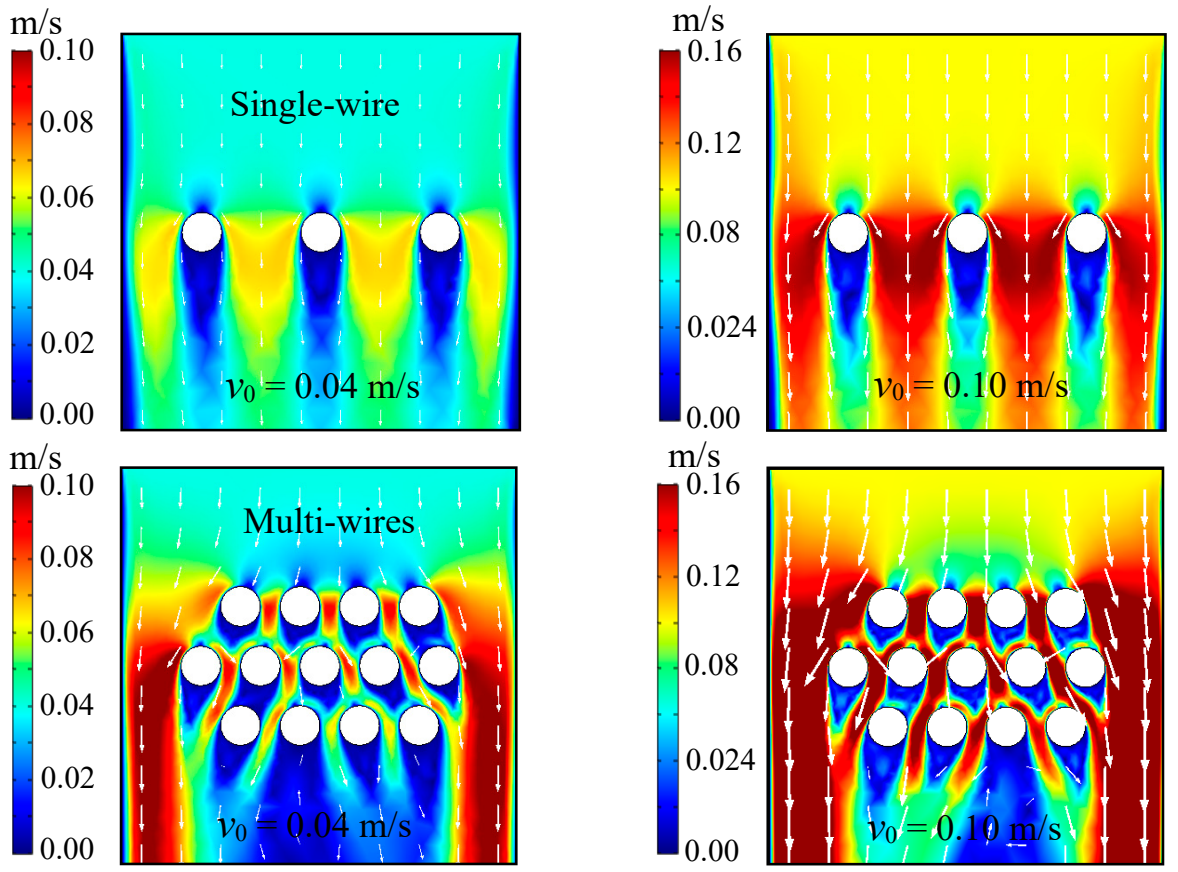

Figure 4. Fluid characteristics around single-wire (above) and multi-wires (below).

\section{Experimental}

\subsection{Cyclic Pilot-Scale Pulsating HGMS Separator}

A SLon-100 cyclic pilot-scale pulsating HGMS separator was used for the present investigation. This separator is fed periodically and its detailed description on the separation mechanism was early reported by Chen et al. [5]. When the separator was operated, via feed box the slurry was fed through the magnetic wire located in the separating zone of the separator, with magnetic particles captured onto the wires and non-magnetic particles flowing out of the zone. When a batch of feed was finished, the energizing current was switched off and the magnetic particles captured onto the wires were washed out to get a magnetic product.

\subsection{Description of Material}

A typical ilmenite ore assaying $13.04 \% \mathrm{TiO}_{2}$ was used for the capture investigation, and the volume magnetic susceptibility of ilmenite particles in the ore is $2.57 \times 10^{-3}$. The material is less than $0.15 \mathrm{~mm}$ and $56.73 \%$ of the material is smaller than $0.074 \mathrm{~mm}$.

\subsection{Method}

Magnetic Capture Analysis (MCA) method [15] was used to investigate the capture selectivity of single wires. As shown in Figure 5, in the method circular holes were regularly drilled in two non-magnetic plates, which were used for inserting magnetic wires. In the investigations, the wires were inserted in the plates at a sufficiently large spacing of $6 \mathrm{~mm}$ to avoid the magnetic coupling effect between the wires.

In this investigation, $\underline{3}$ wires were used in the MCA method, and the material was fed into the separating zone at two different feed modes, to achieve the magnetic capture of wire to particles under ideal and actual conditions, as shown in Figure 6. In the actual condition, $40 \mathrm{~g}$ material was fast fed into the separating zone within $10 \mathrm{~s}$, and was maintained in the zone for a sufficiently long capturing time of $4 \mathrm{~min}$. In the ideal condition, $40 \mathrm{~g}$ material was uniformly divided into 8 fractions, and each fraction was slowly fed into the separating zone within $10 \mathrm{~s}$ and maintained for $4 \mathrm{~min}$; after that, another fraction was fed after $10 \mathrm{~s}$ interval, until all material was fed. 


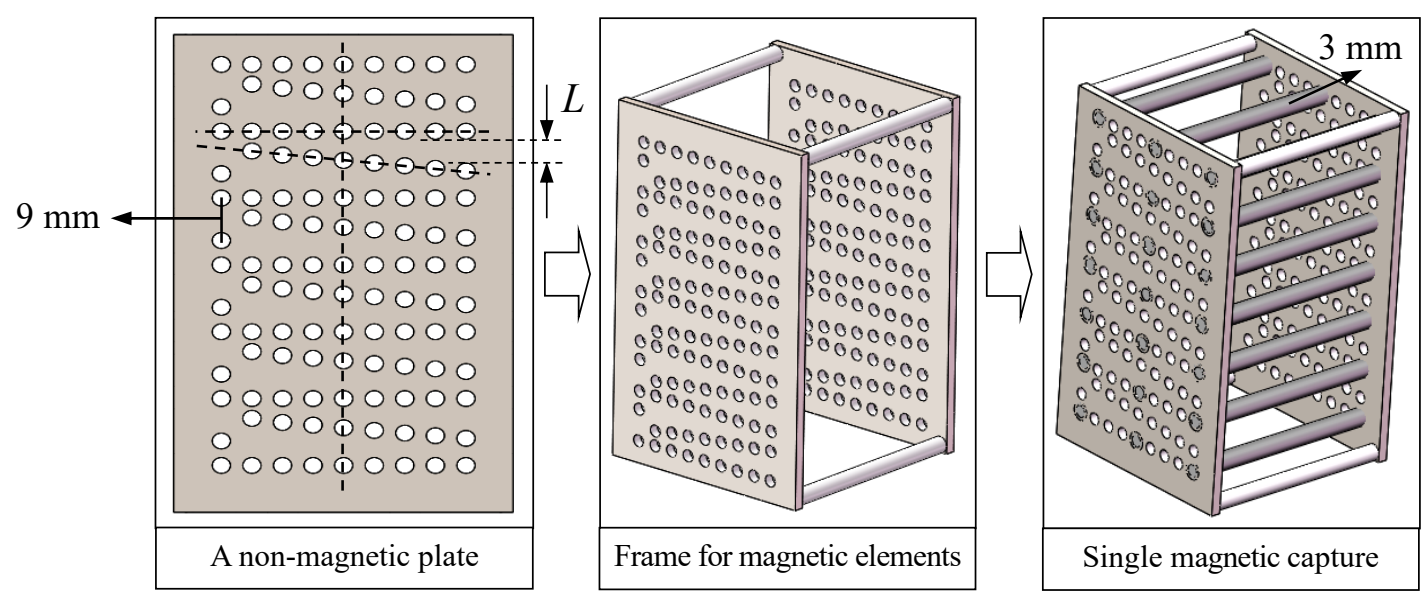

Figure 5. Schematic diagram on operational principle of Magnetic Capture Analysis (MCA) method.

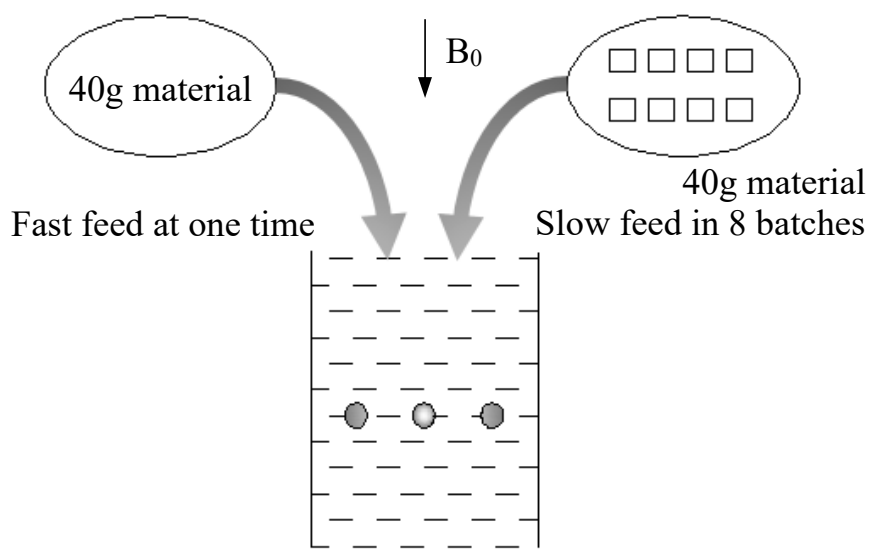

Figure 6. Fast (actual condition) and slow (ideal condition) feed models for material into separating zone.

The capture selectivity of multi-wires was investigated using a real matrix, under the exact same operating conditions as those of single wires. But for this investigation, $200 \mathrm{~g}$ of material was fast fed into the separating zone within $30 \mathrm{~s}$, and the material was maintained in the zone for $8 \mathrm{~min}$.

For all the investigations, 2- and 3-mm diameter magnetic wires were used, and the slurry flows through the separating zone at a velocity of $4.0 \mathrm{~cm} / \mathrm{s}$. In the investigations, the $\mathrm{TiO}_{2}$ grade of capture products and their mass weight for unit length of wire $(\mathrm{g} / \mathrm{cm})$ were respectively used to evaluate the capture selectivity and capture capability of wires.

\section{Results and Discussion}

\subsection{Effect of Magnetic Induction on Capture Selectivity of Single-Wire}

Controlling the pulsating frequency of slurry at $180 \mathrm{r} / \mathrm{min}$, i.e., slurry moving up and down 180 times per minute in the separating zone, and the particle size of $76.56 \%$ below $0.074 \mathrm{~mm}$, the magnetic capture selectivity of single wires, under the slow and fast feed modes as shown in Figure 6, was comparatively investigated, with magnetic induction increasing from $0.4 \mathrm{~T}$ to $1.4 \mathrm{~T}$. From Figure 7, for both the two feed-modes, the $\mathrm{TiO}_{2}$ grade of captured ilmenite products was decreased with increase in the magnetic induction. However, it is obvious that the decrease in the $\mathrm{TiO}_{2}$ grades from the fast feed are much more significantly than that from the slow feed, particularly after $0.8 \mathrm{~T}$.

The most important discovery in this investigation is that the wires in the slow feed mode have achieved higher capture selectivity than that in the fast feed mode, and the experimental results are 
consistent with the theoretical analyses afore. For instance, at the magnetic induction of $0.8 \mathrm{~T}$, the $\mathrm{TiO}_{2}$ grade of capture product from the $3 \mathrm{~mm}$ wire under the slow feed condition reached as high as $36.78 \%$, while it was only $28.32 \%$ for the fast feed. According to the theoretical discussions, in the actual condition (fast feed) non-magnetic particles would be easily entrained into the captured deposits under the action of interactions between particles, so that the capture selectivity of the wire in the actual condition is much lower than that from the ideal condition (slow feed). In addition, in the actual condition, the particles were captured on the wire surface in multi-layers, due to the fact that the particles are fast and massively fed into the separating zone. These multi-layer particles would form a dense deposit on the wire surface under the action of strong magnetic force and the interactions between particles, preventing the entrained particles to be released from the deposit and subsequently reducing the capture selectivity of the wire.
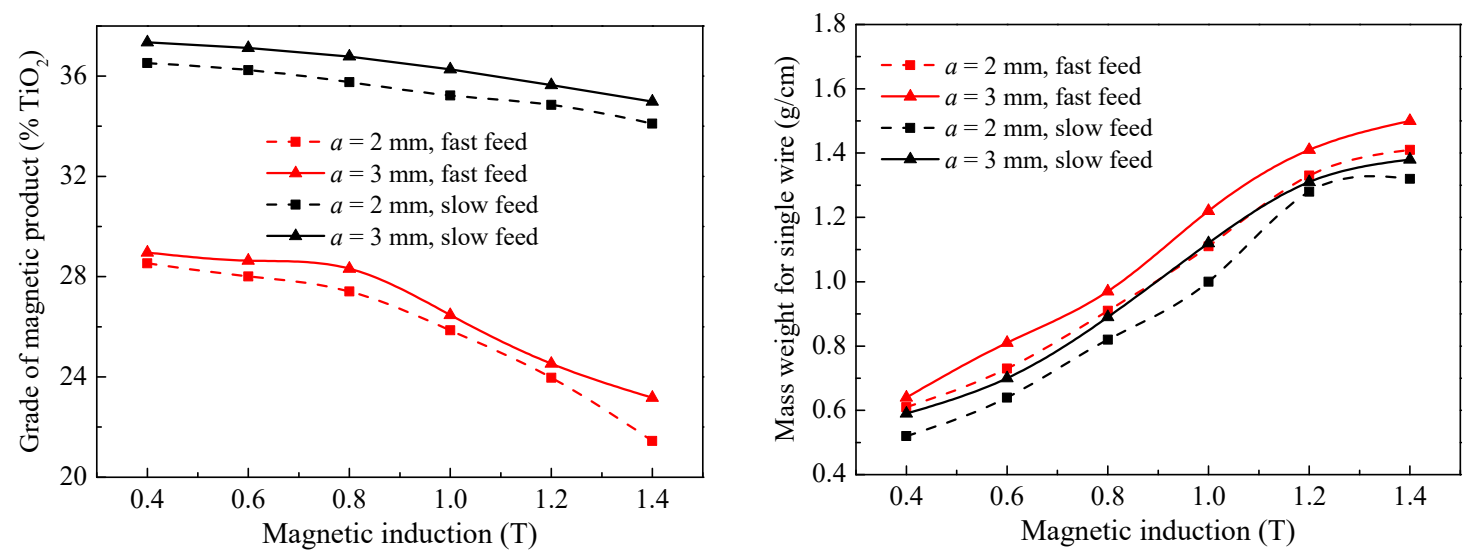

Figure 7. Effect of magnetic induction on capture selectivity (left) and capture capacity (right) of single wire with different feed modes.

It was found that the selective difference between the actual and ideal conditions increased with increase in the magnetic induction. Such an increase in the difference may be mainly due to the fact that under the high magnetic induction, non-magnetic particles more easily collided with the wire in the actual condition, as shown in Figure 3, and so it is much more difficult for the entrained particles to release from the magnetic deposits in the higher induction.

Also, the mass weight of capture product increased with increase in the magnetic induction, and the wires in the ideal condition have achieved slightly higher capture mass weight than that from the wires in the actual condition. It should be noted that this investigation aims to investigate the capture selectivity of wires in high gradient magnetic field, and therefore only the $\mathrm{TiO}_{2}$ grade of capture product was considered in the following investigations.

\subsection{Effect of Pulsating Frequency on Capture Selectivity of Single-Wire}

It is well known that the pulsation of slurry produces a relaxed effect to particles in the slurry, which improves the capture selectivity of magnetic wires. As shown in Figure 8, similar to the effect of magnetic induction as discussed above, the wires from the slow and fast feed models have basically the same capture trend while the pulsating frequency were gradually increased; but again, the effect of pulsating frequency on the capture selectivity of wires from the slow feed is much more gentle than that from the wires in fast feed. It was also found that the wires in the slow feed mode achieved a higher capture selectivity than that from the wires of fast feed mode.

As discussed afore, the entrainment of non-magnetic particles in the magnetic deposits may be mainly resulted from the interactions between particles, and the hydrodynamic force is the main force competing the interaction in the capture process. As the pulsation of slurry increases, the hydrodynamic force acting onto the particles weakens the interaction and facilitates the release of non-magnetic particles from magnetic deposits, thereby improving the capture selectivity of wires. But in the slow 
feed, non-magnetic particles are rarely entrained into the captured deposits and the magnetic wires achieved a high capture selectivity, so that the capture selectivity of wires in the slow feed is not so sensitive to the pulsating frequency.

However, it is noted that the entrainment of non-magnetic particles could not be completely eliminated in the pulsating slurry. From Figure 8, even at high pulsating frequency, the $\mathrm{TiO}_{2}$ grade of magnetic product from the fast feed mode is still lower than that from the slow one.

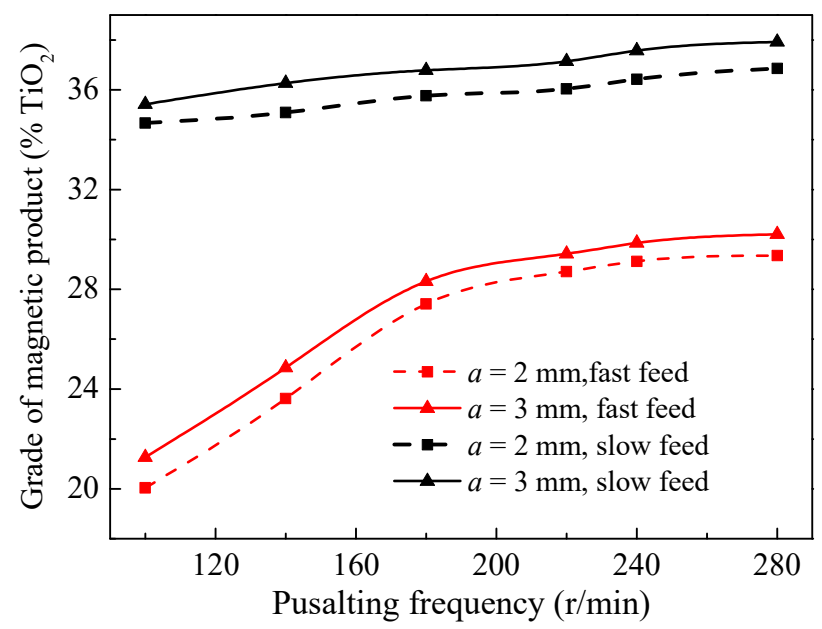

Figure 8. Effect of pulsating frequency on capture selectivity of single-wire with different feed modes.

\subsection{Effect of Particle Size on Capture Selectivity of Single-Wire}

It is well known that the particle size determines the liberation degree of material and so that produces significant effect on the capture selectivity of magnetic wire, as shown in Figure 9. It should be noted that this effect is investigated under magnetic induction of $0.8 \mathrm{~T}$ and pulsating frequency of $180 \mathrm{r} / \mathrm{min}$. From Figure 9, for both the two feed-modes, the $\mathrm{TiO}_{2}$ grades of captured deposits were increased with decrease in the particle size of feed material from $56.73 \%$ to $76.56 \%$ below $0.074 \mathrm{~mm}$, and they approached the maximum values with further decrease in the particle size to $91.23 \%$ below $0.074 \mathrm{~mm}$. It was also found that the wires in the slow feed mode achieved a higher $\mathrm{TiO}_{2}$ grade than that from the wires in fast feed mode, and the grade difference between the fast and slow feeds increased with decrease in the particle size.

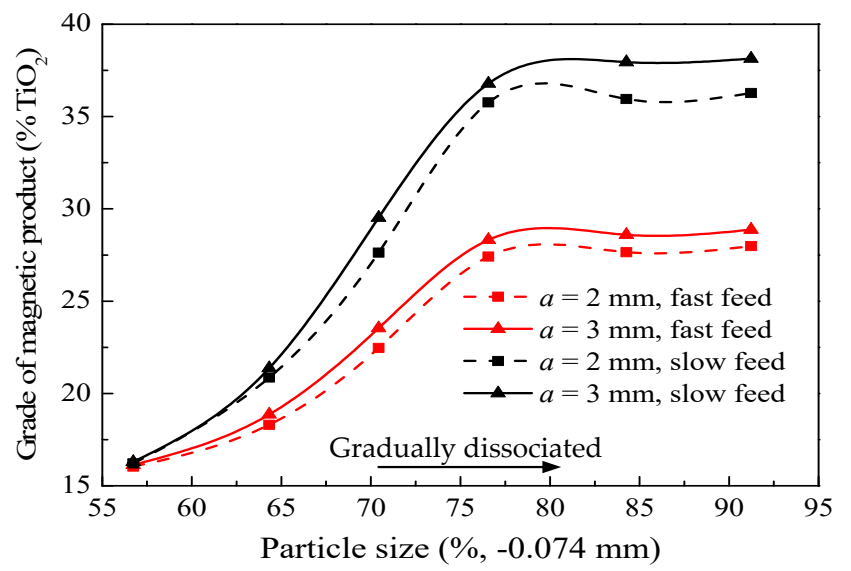

Figure 9. Effect of particle size on capture selectivity of single wire with different feed modes.

For large particle size, the material was not fully dissociated, and then many intergrowth minerals combining magnetic and non-magnetic components may be captured on the wire surface, reducing the $\mathrm{TiO}_{2}$ grade of captured deposits. With decrease in the particle size, the intergrowths would be 
gradually liberated, which reduces the number of intergrowth minerals in the magnetic products and improves their $\mathrm{TiO}_{2}$ grades. However, in the fast feed mode, many non-magnetic particles would be easily entrained into the magnetic deposits, as clearly described above, so that with decreasing particle size, the increase in the $\mathrm{TiO}_{2}$ grades of magnetic products in the slow feed is not so significant as that in the fast feed.

\subsection{Comparation of Capture Selectivities between Single Wire and Multi-Wires}

As mentioned afore, the multi-wires have different capture characteristics from the single wire. Thus, in this section, the capture selectivity of single wire and multi-wires were comparatively investigated, with magnetic induction increasing from $0.4 \mathrm{~T}$ to $1.4 \mathrm{~T}$, as shown in Figure 10 . It is noted that the material was fed in the fast feed mode as mentioned afore. From Figure 10, the single wire and multi-wires produced two similar sets of capture selectivity, and the $\mathrm{TiO}_{2}$ grades of magnetic products reduced with increase in the magnetic induction. However, it was found that the multi-wires produced much lower $\mathrm{TiO}_{2}$ grades of magnetic products than those from the single wire, and the capture selectivity of multi-wires was much more sensitive to variations in the magnetic induction.

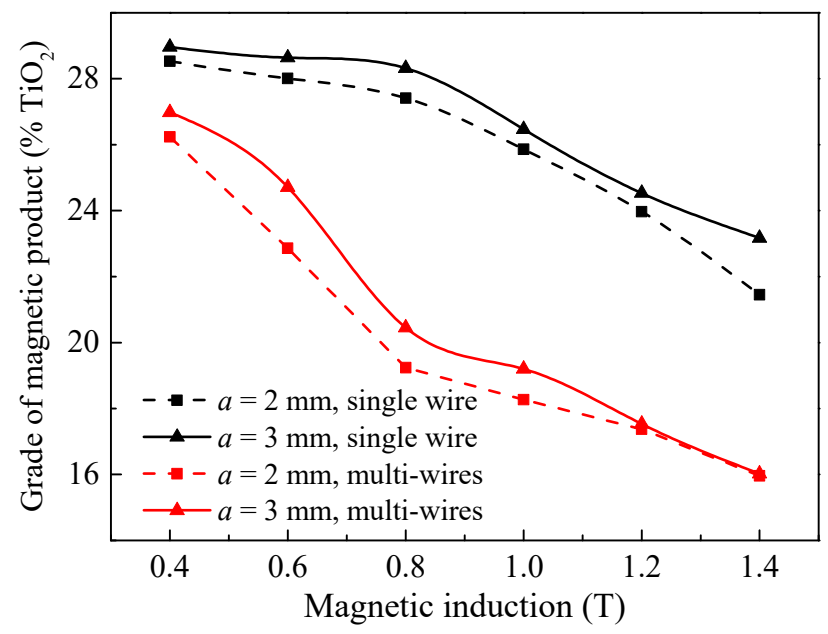

Figure 10. Capture selectivity of single wire and multi-wires.

The reduced capture selectivity of the multi-wires is mainly resulted from the poor flowability of slurry in the multi-wires, as shown in Figure 4. In addition, in the multi-wires, after an entrained particle is released from magnetic deposits on the wire surface, the particle may collide with other adjacent wires, increasing the probability of non-magnetic particle entraining into capture deposits. Therefore, the separating selectivity in the actual HGMS process with multi-wires is much lower than that from the ideal one.

\section{Conclusions}

1. The motion trajectories of a few of particles in the ideal HGMS condition is significantly different from those of a lot of particles in the actual HGMS condition, as the interactions between particles and those between particles and slurry have their significant effects on the trajectories. In addition, the captured particles form a compact deposit on the wire surface due to the interactions between the particles, preventing entrained particles to be released from the deposit; therefore, non-magnetic particles would be more easily entrained into the deposit in the actual HGMS condition than those in the ideal HGMS condition. The flowability of slurry in the multi-wires is much weaker than that through a single-wire.

2. The experimental results are consistent with the theoretical analyses. The wires in slow feed mode produced a higher capture selectivity than that in fast feed mode, and their difference in the selective capture increased with increase in the magnetic induction and decrease in the pulsating 
frequency. The multi-wires produced much lower $\mathrm{TiO}_{2}$ grades of magnetic products than those from the single wire, as they produce different effects on the flowability of slurry.

3. The selective capture of magnetic wire to magnetic particles could be improved in a pulsating slurry, but the entrainment of non-magnetic particles in the magnetic deposits on wire surface could not be fully eliminated.

This investigation provides a beneficial understanding on the selective capture of magnetic wires to magnetic particles in a HGMS process.

Author Contributions: Methodology, J.Z. and L.C.; validation, J.Z. and F.Y.; formal analysis, J.Z.; investigation, J.Z.; resources, L.C. and X.T.; data curation, J.Z. and F.Y.; writing — original draft preparation, J.Z.; writing - review and editing, J.Z. and L.C.; supervision, L.C. and X.T.; project administration, L.C. and X.T.; funding acquisition, L.C. and X.T.

Funding: This research was funded by National Natural Science Foundations of China (Grant No. 51874152) and the Key Program for Applied Basic Research of Yunnan Province (Grant No. 2016FA051).

Conflicts of Interest: The authors declare no conflict of interest.

\section{References}

1. Padmanabhan, N.; Sreenivas, T. Process parametric study for the recovery of very fine size uranium values on super-conducting high gradient magnetic separator. Adv. Powder Technol. 2011, 22, 131-137. [CrossRef]

2. Chen, L.; Xiong, D.; Huang, H. Pulsating high-gradient magnetic separation of fine hematite from tailings. Miner. Metall. Proce. 2009, 26, 163-168. [CrossRef]

3. Chen, L.; Liao, G.; Qian, Z.; Chen, J. Vibrating high gradient magnetic separation for purification of iron impurities under dry condition. Int. J. Miner. Process. 2012, 130, 136-140. [CrossRef]

4. Zheng, X.; Wang, Y.; Lu, D. Study on capture radius and efficiency of fine weakly magnetic minerals in high gradient magnetic field. Miner. Eng. 2015, 74, 79-85. [CrossRef]

5. Chen, L.; Liu, W.; Zeng, J.; Ren, P. Quantitative investigation on magnetic capture of single wires in pulsating HGMS. Powder Technol. 2017, 313, 54-59. [CrossRef]

6. Xiong, D. SLon magnetic separator applied in the ilmenite processing industry. Phys. Sep. Sci. Eng. 2004, 13, $119-126$.

7. Zeng, W.; Xiong, D. The latest application of SLon vertical ring and pulsating high-gradient magnetic separator. Miner. Eng. 2003, 16, 563-565. [CrossRef]

8. Xiong, D.; Liu, S.; Chen, J. New technology of pulsating high gradient magnetic separation. Int. J. Miner. Process. 1998, 54, 111-127. [CrossRef]

9. Ren, P.; Chen, L.; Liu, W.; Shao, Y.; Zeng, J. Comparative investigation on magnetic capture selectivity between single wires and a real matrix. Results Phys. 2018, 8, 180-183. [CrossRef]

10. Li, W.; Han, Y.; Xu, R.; Gong, E. A preliminary investigation into separating performance and magnetic field characteristic analysis based on a novel matrix. Minerals 2018, 8, 94. [CrossRef]

11. Zheng, X.; Wang, Y.; Lu, D. Particle capture efficiency of elliptic cylinder matrices for high-gradient magnetic separation. Sep. Sci. Technol. 2016, 51, 2090-2097. [CrossRef]

12. Zeng, J.; Tong, X.; Ren, P.; Chen, L. Theoretical description on size matching for magnetic element to independent particle in high gradient magnetic separation. Miner. Eng. 2019, 135, 74-82. [CrossRef]

13. Zheng, X.; Wang, Y.; Lu, D. Study on buildup of fine weakly magnetic minerals on matrices in high gradient magnetic separation. Physicochem. Probl. Miner. Process. 2017, 53, 94-109.

14. Svoboda, J. A realistic description of the process of high-gradient magnetic separation. Miner. Eng. 2001, 14, 1493-1503. [CrossRef]

15. Lu-Zheng, C.; Wen-Bo, L.; Qing-Fei, X.; Jian-Wu, Z.; Xiong, T.; Zheng, Y.-M. An Experimental Method for Magnetic Capture Analysis of Single Magnetic Wires. China Patent No. 20161057 4053.3, 21 June 2016. (In Chinese).

(C) 2019 by the authors. Licensee MDPI, Basel, Switzerland. This article is an open access article distributed under the terms and conditions of the Creative Commons Attribution (CC BY) license (http://creativecommons.org/licenses/by/4.0/). 